\title{
Complete Coating of Underlying Pt Electrodes by Electrochemical Reduction of Graphene Oxide
}

Jason A. Bennett*, Issaka B. Agbere, and Matthew Moesta

School of Science, Penn State Erie, The Behrend College, 4205 College Drive, Erie, Pennsylvania 16563, USA

${ }^{*}$ Corresponding author Tel.: 1-814-898-6123; Fax: 1-814-898-6213

E-mail address: jab95@psu.edu (J. A. Bennett) 


\begin{abstract}
Graphene oxide electrochemically reduced from aqueous and nonaqueous graphene oxide suspensions was compared. The protocol that utilized the nonaqueous suspension resulted in a more reproducibly complete covering of the underlying Pt electrode while still retaining its electrochemical integrity. The hydrogen evolution reaction was found to be detrimental to achieving complete electrode coverage from the aqueous suspension due to $\mathrm{H}_{2}$ bubbles physically dislodging previously reduced graphene oxide. However, since this parasitic reaction does not proceed as readily in organic solvents such as DMF, the nonaqueous reduction was able to form a complete layer once the aqueous suspension was drop-casted and dried on the Pt electrode. The graphene oxide-coated electrodes resulting from both aqueous and nonaqueous protocols were compared using electrochemistry, SEM, AFM, and XPS. Overall, the sample produced from the nonaqueous suspension possessed a significantly rougher morphology and was determined to possess a higher amount of reduced carbon than that produced using the aqueous suspension.
\end{abstract}

Keywords: graphene, graphene oxide, ERGO, electrocatalyst support, reduced graphene oxide 


\section{Introduction}

Graphene is a two-dimensional $\mathrm{sp}^{2}$-hybridized carbon material that has many attractive physical and chemical properties, which have increased its popularity in recent years for a variety of applications. The reduction of graphene oxide (GO) is one of the most promising routes for achieving graphene at a low cost and high yield, even though the process only partially restores the properties of pristine graphene. While there are several methods for reducing GO such as chemical, thermal, and photocatalytic methods, electrochemical reduction has been regarded as the most attractive method due to the absence of reducing agents, which are harmful to both humans and the environment, and can contaminate the final product.[1] and references therein

Reduced graphene oxide produced via the electrochemical reduction of exfoliated graphite oxide has attracted much attention due to its relative simplicity, speed, potential for controllability, and benign environmental impact.[2, 3] There has been a lot of research in recent years understanding the fabrication and characterization of electrochemically reduced graphene oxide (ERGO) in order for it to be used for various applications.[3-16] Specifically, there have been several studies using ERGO as either a sensing material or electrocatalyst support for detecting various analytes.[15, 17-19]

There is a lot of research on ERGO, however most of these studies focus on reducing the GO on to a glassy carbon electrode, and of those that do focus on depositing ERGO onto metallic surfaces, very few ascertain whether the underlying electrode surface is completely covered by the ERGO layer. The exposure of the underlying metal electrode can severely impact the observed electrochemistry, and specifically hinder the selectivity in 
sensing applications. Moreover, the complete coverage of the underlying metal electrode is quite difficult to achieve, especially when reducing from an aqueous suspension of exfoliated graphite oxide. This is due to the fact that, in order to get the ERGO to precipitate on to the electrode surface, sufficiently negative potentials must be applied. This means that the GO reduction process competes with the reduction of the suspension solvent (i.e., hydrogen evolution).[2, 7] The competition between these two reduction processes is much more problematic in the case of metal electrodes. To our knowledge, only Guo et al. have utilized electrochemistry, with its abilities to easily identify pinholes in the ERGO layer, to verify complete surface coverage of a metal electrode.[5]

While most of the literature focuses on ERGO fabricated from aqueous solutions, two have involved ERGO fabricated from nonaqueous solutions, which will minimize the breakdown of the suspension solvent. Harima et al. deposited ERGO onto a variety of electrode surfaces, including Pt, by initially drop-casting $50-\mu \mathrm{L}$ of a $2.0 \mathrm{mg} / \mathrm{mL}$ aqueous GO suspension. After drying, the electrode was placed in an organic electrolyte/solvent solution (without GO) and the dried GO was successfully reduced at given potentials.[6] Kauppila and coworkers followed a similar protocol to determine that the reduction in nonaqueous solvents requires a more negative potential compared to aqueous solvents, and that slightly different ERGO layers are fabricated.[8] However, neither study assessed the surface coverage of the ERGO layer.

The work presented herein proposes a new protocol to achieve reproducibly complete ERGO layers on Pt. The new protocol involves electrochemically reducing GO 
from a nonaqueous (DMF) GO suspension (ERGO ${ }_{\mathrm{DMF}}$ ) and compares it to that deposited from a typical aqueous $\mathrm{GO}$ suspension ( $\left.\mathrm{ERGO}_{\mathrm{Aq}}\right)$. The modified-electrodes are compared electrochemically to determine relative surface coverage and electrochemical activity. While developing this protocol on Pt is not relevant for most practical applications, it was chosen here because Pt is a very electrochemically active metal that will easily reveal any pinholes in the ERGO layer using the $2 \mathrm{H}^{+} / \mathrm{H}_{2}$ redox couple. The physical and chemical properties of the ERGO samples are also assessed using SEM, AFM, and XPS techniques.

Our long-term goal is to utilize the ERGO as an electrocatalyst support material for sensing applications; this requires that the underlying metallic surface be completely coated so that it does not interfere with the sensing selectivity. However, considering the interest in utilizing reduced graphene oxide in various fields including energy (e.g., fuel cells, solar cells, and storage) and electronic (e.g., supcapacitors, batteries, and transistors) areas, this new protocol is expected to be attractive for adaptation to other applications. For example, the new protocol is faster and yields a more reproducible coating than using just an aqueous suspension. This should allow for better control and possibly help facilitate mass production. Additionally, the high number of cyclic voltammetric cycles required to achieve a complete coating in an aqueous GO suspension is expected to produce ERGO with more defects within its structure.[11] The new protocol requires substantially fewer cycles to achieve a complete layer. Finally, the nonaqueous suspension allows for more negative potential limits, which is expected to remove more oxygen-containing functional groups. This should help restore the $\mathrm{sp}^{2}$ character, increase conductivity, and result in stronger and more compact coatings. 


\section{Experimental}

\subsection{Graphite Oxide Synthesis and Suspension Preparation}

All chemicals were reagent grade and used as received. The graphite oxide was synthesized using a modified version of Marcano's method.[20] Briefly, 360 mL of 95-98\% $\mathrm{H}_{2} \mathrm{SO}_{4}$ and $40 \mathrm{~mL}$ of $85 \% \mathrm{H}_{3} \mathrm{PO}_{4}$ were added into a 1-L Erlenmeyer flask containing $25 \mathrm{~g}$ of $\mathrm{KMnO}_{4}$ and $5 \mathrm{~g}$ of graphite flakes (Aldrich). The mixture was then stirred overnight (no more than 20 hours) while being held at a temperature of $50^{\circ} \mathrm{C}$. The heat was turned off and the mixture was allowed to cool to room temperature. At this point the mixture became a brownish thick paste. Next, approximately $400 \mathrm{~mL}$ of ice was added, and as the ice melted, the Erlenmeyer flask became hot as the mixture turned purplish in color. Once all the ice had melted (20 - 30 minutes), $30 \mathrm{~mL}$ of $30 \% \mathrm{H}_{2} \mathrm{O}_{2}$ was added drop wise (carefully), while stirring, using a separatory funnel in order to prevent the solution from bubbling over. The temperature of the mixture increased during this addition. The solution was stirred for at least 15 more minutes resulting in a yellowish mixture. This mixture was sifted for an hour or two using a Retsch 300- $\mu$ m sieve. What remained on the sieve was then scraped off into a beaker and, after cleaning the sieve, was sifted again. The process was repeated one or two more times, and usually left to stand overnight in order to obtain more filtrate.

The filtrate was then transferred into three 200 -mL polyethylene centrifuge bottles and centrifuged for $1 \mathrm{~h}$ at $5000 \mathrm{rpm}$ using a Sorvall RC 5C Plus Centrifuge (rotor 10). The supernatant was decanted, and the precipitate was washed sequentially washed with 200 
mL each of ultra-pure water, $35 \% \mathrm{HCl}$, ethanol, and again with ethanol. The suspension was sifted, centrifuged, and had the supernatant decanted between each washing. Finally the solid material left was washed with $200 \mathrm{~mL}$ of diethyl ether and then vacuum filtered using multiple $0.5-\mu \mathrm{m}$ hydrophobic filters. The GO had a brownish color and was dried in a desiccator under vacuum.

Two different GO suspensions were prepared. The aqueous suspension was prepared by exfoliating $15 \mathrm{mg}$ of GO in $20 \mathrm{~mL}$ of ultra-pure water $(0.75 \mathrm{mg} / \mathrm{mL})$ by ultrasonicating for 3 hours. Then $0.5 \mathrm{~g} \mathrm{NaCl}$ was added to give a concentration of $0.4 \mathrm{M}$, and the suspension was sonicated for 30 additional minutes. The nonaqueous GO suspension similarly used $15 \mathrm{mg}$ of GO exfoliated in $20 \mathrm{~mL}$ of $99.8 \%$ anhydrous $\mathrm{N}, \mathrm{N}-$ dimethylformamide solvent (DMF; Sigma-Aldrich in Sure/Seal ${ }^{\mathrm{TM}}$ ) by ultrasonicating for 3 hours. Then $0.66 \mathrm{~g}$ of tetrabutylammonium tetrafluoroborate (TBATFB) was added to yield a $0.1 \mathrm{M}$ concentration.

\subsection{Electrochemical Reduction}

Platinum macroelectrodes $(\mathrm{d}=2.0 \mathrm{~mm})$ were polished sequentially using an Alpha Alumina powder slurry from 1.0, 0.3 and $0.05 \mu \mathrm{m}$ grits for 7 minutes each and ultrasonicated for 7 minutes after each grit. The polished Pt electrode was then placed in deoxygenated $0.5 \mathrm{M} \mathrm{H}_{2} \mathrm{SO}_{4}$ and electrochemically activated using the following cyclic voltammetric sequence: 1 cycle from $-0.25 \mathrm{~V}$ to $1.3 \mathrm{~V}$ at $100 \mathrm{mV} / \mathrm{s} ; 1$ cycle from $0.2 \mathrm{~V}$ to 2.1 $\mathrm{V}$ to $-0.5 \mathrm{~V}$ to $0.2 \mathrm{~V}$ at $100 \mathrm{mV} / \mathrm{s}$.; 25 cycles from $0.2 \mathrm{~V}$ to $1.25 \mathrm{~V}$ to $-0.25 \mathrm{~V}$ at $100 \mathrm{mV} / \mathrm{s}$ and 
at to end the process, the Pt electrode was held at $0.2 \mathrm{~V}$ for $60 \mathrm{~s}$, and rinsed with ultra-pure water

Both reduction protocols utilized an initial $10-\mu \mathrm{L}$ aliquot of $\mathrm{N}_{2}$-saturated aqueous GO suspension drop-casted on the Pt electrode and allowed to dry at $100^{\circ} \mathrm{C}$ for $10 \mathrm{~min}$. The electrode was then placed in one of the GO suspensions with a carbon rod (auxiliary), and either a $\mathrm{Ag} / \mathrm{AgCl}(3 \mathrm{M} \mathrm{NaCl}$; aqueous suspension) reference or a $\mathrm{Ag}$ wire quasireference (QRE; nonaqueous suspension) electrode, respectively. The reference potential of the Ag QRE was assessed using ferrocene in 0.1 M TBATFB/DMF, which yielded an Eo of $709 \mathrm{mV}$ at a clean Pt electrode. The aqueous reduction was performed by cycling the potential 100 times from 0.4 to $-1.45 \mathrm{~V}$ vs. $\mathrm{Ag} / \mathrm{AgCl}$, at a scan rate of $50 \mathrm{mV} / \mathrm{s}$ while stirring the solution and having $\mathrm{N}_{2}$ gas bubbled through the solution as far away from the electrode as possible. The nonaqueous reduction was performed in a similar manner, but by cycling the potential in the DMF suspension 25 times between $0.4 \mathrm{~V}$ and $-1.6 \mathrm{~V}$ vs. Ag QRE. After deposition, the modified-electrode was carefully immersed in the respective solvent (ultrapure water or DMF) to rinse it off without dislodging the deposited ERGO layer and allowed to dry in air overnight. The electrodes will be denoted as either $\mathrm{ERGO}_{\mathrm{Aq}}$ or $\mathrm{ERGO}_{\mathrm{DMF}}$, respectively.

\subsection{Electrode Characterization}

The $\mathrm{ERGO}_{\mathrm{Aq}}$ and $\mathrm{ERGO}_{\mathrm{DMF}}$ electrodes were electrochemically characterized using 5 $\mathrm{mM} \mathrm{HCl}, 1 \mathrm{mM} \mathrm{Fe}(\mathrm{CN}) 6^{3-/ 4-}$, and $1 \mathrm{mM} \mathrm{Ru}\left(\mathrm{NH}_{3}\right)_{6}{ }^{3+/ 2+}$ all dissolved in either 0.5 or $1 \mathrm{M} \mathrm{KCl}$. Physical characterization was performed using both a Bruker Icon Atomic Force 
Microscope in Peak Force Tapping Mode and a Zeiss EVO HD15 Scanning Electron

Microscope at $20 \mathrm{kV}$ accelerating voltage and a $5 \mathrm{~mm}$ working distance. Additionally, X-ray photoelectron spectroscopy was performed using a Kratos Axis Ultra Spectrometer with monochromatic Al-Ka X-rays. Spectra were obtained in 2 locations on each electrode and were charge-corrected to C-C @ $285 \mathrm{eV}$. Representative data are presented.

\section{Results}

\subsection{Electrode Modification}

Two protocols for the electrochemical reduction of graphene oxide (ERGO) onto Pt electrodes were compared in order to determine which method would consistently yield a fully coated underlying surface. For comparison, both protocols involved initially dropcasting a $10-\mu \mathrm{L}$ aliquot of the aqueous GO suspension on to the clean Pt electrode surface and allowing it to dry at $100{ }^{\circ} \mathrm{C}$ for 10 min prior to placing it in either the aqueous or DMF GO suspension for electrochemical reduction. The presence of this initial aqueous drop prior to electrochemical reduction showed no effect on the reduction in aqueous media but had a profound impact on the nonaqueous reduction, as will be discussed in section 3.2 . Therefore, to maintain consistency, this initial 10- $\mu \mathrm{L}$ aqueous drop was included in both reduction protocols. The first protocol reduced the GO in a traditional aqueous electrolyte $(\mathrm{NaCl})$ suspension, by placing the dried electrode back in to the aqueous $\mathrm{GO}$ suspension and cycling the potential 100 times between $300 \mathrm{mV}$ and $-1450 \mathrm{mV}$ at a rate of $50 \mathrm{mV} / \mathrm{s}$ $\left(E G_{A q}\right)$. The second protocol involved placing the dried electrode in a nonaqueous GO suspension of TBATFB/DMF and cycling the potential 25 times between $300 \mathrm{mV}$ and -1600 $\mathrm{mV}$ vs. Ag QRE (ERGO $\mathrm{DMF}_{\mathrm{DF}}$ ). Representative voltammetric $i-E$ curves for both reduction 
protocols are shown in Figure 1. Figure 1A shows an enlarged region of the $11^{\text {st }}, 25^{\text {th }}, 50^{\text {th }}$, $75^{\text {th }}$, and $100^{\text {th }}$ cycle for the $\mathrm{ERGO}_{\mathrm{Aq}}$ reduction. The first cycle shows a marked increase in reduction current at about $-250 \mathrm{mV}$, associated with the onset of hydrogen evolution at the Pt electrode surface. The magnitude of this current continues to increase beyond the displayed area of the plot in Figure 1A. As the cycle number increases, the onset potential for hydrogen evolution shifts cathodically to about $-400 \mathrm{mV}$. This is followed on the reverse scan by an oxidation peak, which also shifts to more negative potentials with cycle number. This oxidation is attributed to hydrogen desorption on the exposed Pt. The onset of $\mathrm{H}_{2}$ evolution produced bubbles of hydrogen gas, that were visible on the electrode surface during the potential cycling, which physically dislodged previously reduced GO. A minimum of 100 cycles was determined, using $\mathrm{HCl} / \mathrm{KCl}$ electrochemistry (discussed in section 3.2), to be required in order to achieve a complete ERGO layer.

In the interest of avoiding the formation of $\mathrm{H}_{2}$ bubbles, and hopefully result in a more efficient reduction, the use of a nonaqueous TBATFB/DMF GO suspension was investigated. DMF was chosen as a solvent as it has been shown to form a stable GO suspension while also serving as a good electrochemical solvent.[21,22] As with the $\mathrm{ERGO}_{\mathrm{Aq}}$ sample, a $10-\mu \mathrm{L}$ drop of the aqueous $\mathrm{GO}$ suspension was placed on the electrode surface and allowed to dry prior to electrochemical reduction. The potential was then cycled in the DMF GO suspension as shown in Figure 1B. The first cycle (black) shows two reduction signals at $c a .-275 \mathrm{mV}$ and $-725 \mathrm{mV}$, respectively. The peak at $-275 \mathrm{mV}$ is attributed to the reduction of the dried aqueous GO film, as that peak was not present when the aqueous suspension was not drop-casted on the surface, nor is any oxidation peak 
observed on the reverse scan. The peak at $-725 \mathrm{mV}$ is attributed to the reduction of the GO in the DMF suspension. The magnitude of the reduction current initially decreases due to the covering of the Pt electrode, which has a higher double-layer capacitance compared to the ERGO. The magnitude of the reductive current then increases after cycle 5; consistent with an increase in ERGO thickness. The very slight difference between the $20^{\text {th }}$ and $25^{\text {th }}$ cycle suggested a complete ERGO layer was obtained.

There are some marked differences worth noting between the voltammetric $i-E$ curves for the DMF reduction and that from the aqueous reduction in 1A. First, as expected, there was no hydrogen evolution to compete with and hence no bubbles were visibly formed on the electrode surface in the DMF suspension. The potential could likely have been carried even farther negative, but in the interest of comparing the two methods, the negative limit was chosen to be close to that of the aqueous reduction. The second noticeable difference is that $i-E$ curves in the nonaqueous reduction are much noisier than those in the aqueous solution, and increases with increasing cycle number. In fact, in order to differentiate the individual cycles as shown in Figure $1 \mathrm{~B}$, the actual data from reduction had to be smoothed in the PSI-Plot data analysis software. This is likely due to the conductivity of the solution between the reduced layers of the GO. The aqueous reduction utilizes a highly conductive $\mathrm{NaCl}$ electrolyte $(\sim 28 \mathrm{mS} / \mathrm{cm})$ that would likely keep conductivity between the layers, despite the increased thickness of the ERGO layer. In comparison, the conductivity of the nonaqueous suspension was measured to be $\sim 4$ $\mathrm{mS} / \mathrm{cm}$ using a Pt conductivity probe. The noise in Figure 1B increases with cycle number suggesting that this is due to the conductivity through the ERGO layer. As will be seen in 
the electrochemical characterization, this noise does not appear to have an adverse effect on the electrochemistry once placed in an aqueous solution.

\subsection{Electrochemical Characterization}

In order to determine whether the underlying Pt electrode was fully coated, cyclic voltammetry was performed in a $5 \mathrm{mM} \mathrm{HCl} / 0.5 \mathrm{M} \mathrm{KCl}$ solution as described by Guo et al.[5] Figure 2 shows representative voltammetric $i-E$ curves for the $\mathrm{HCl} / \mathrm{KCl}$ solution at clean Pt (black), $\mathrm{ERGO}_{\mathrm{DMF}}(\mathrm{red})$ and $\mathrm{ERGO}_{\mathrm{Aq}}$ (blue) electrodes. The inset of Figure 2 shows an enlargement of the $\mathrm{ERGO}_{\mathrm{DMF}}$ and $\mathrm{ERGO}_{\mathrm{Aq}}$ curves to better highlight their differences. The clean Pt electrode exhibits a very reversible redox couple at $-375 \mathrm{mV}$ attributed to the $2 \mathrm{H}^{+} / \mathrm{H}_{2}$ redox reaction. $[5,23,24]$ This is a surface sensitive reaction that is reversible on clean Pt, but becomes increasing irreversible as the underlying Pt electrode is coated with the ERGO layer.[5, 24] It can therefore be used to probe whether the underlying Pt is indeed completely coated by the ERGO layer. As expected, curves for both modifiedelectrodes were void of the reversible $2 \mathrm{H}^{+} / \mathrm{H}_{2}$ redox couple compared to the clean Pt curve. However, as shown in the inset of Figure 2, the $\mathrm{ERGO}_{\mathrm{Aq}}$ electrode still exhibits noticeable reduction current at $c a .-500 \mathrm{mV}$ and some oxidation at $c a .-200 \mathrm{mV}$ indicating that some portion of the underlying Pt electrode is still exposed to the $\mathrm{HCl} / \mathrm{KCl}$ solution. In contrast, the $E_{R G O}{ }_{D M F}$ electrode exhibits no observable redox current indicating complete coverage of the underlying Pt.

To determine whether the full $\mathrm{ERGO}_{\text {DMF }}$ protocol is necessary to achieve a complete ERGO layer, two control deposition experiments were performed. Control 1 attempted to 
reduce the GO onto a clean Pt electrode directly from the DMF GO suspension (without the initial drop-casted aqueous suspension) using the same cyclic voltammetric parameters as described in Figure 1B. This was performed to determine whether the ERGO could be directly deposited from the nonaqueous suspension or if the presence of water impacts the electrode modification. Control 2 utilized a Pt electrode with the drop-casted aqueous GO suspension, but the potential was cycled in the TBATFB/DMF electrolyte solution in the absence of GO. This was similar to protocols used in earlier studies of ERGO from organic solutions. $[6,8]$ This latter control was performed to determine if the coating occurs solely from the reduction of the dried aqueous suspension, or if additional GO is reduced from the DMF GO suspension.

The $\mathrm{HCl} / \mathrm{KCl}$ voltammetric $i-E$ curves for each control are compared in Figure 3 with that from the complete ERGO $\mathrm{DMF}_{\text {DM }}$ protocol. It is evident that only the $\mathrm{ERGO}_{\mathrm{DMF}}$ electrode (black) utilizing the complete protocol exhibits a flat $i-E$ curve. When the aqueous suspension is not drop-casted (Control 1; red), there was a very significant reduction peak at $-450 \mathrm{mV}$ and oxidation at $c a .-250 \mathrm{mV}$. While the redox couple is not as pronounced as on the clean Pt, suggesting that there is some ERGO on the Pt surface, this obviously does not yield complete coverage of the underlying electrode surface. These signals support the necessity of the dried aqueous suspension in the complete protocol as well as implicate the importance of either intercalated water or GO sites on the Pt, in order to facilitate ERGO nucleation/precipitation from the DMF suspension. The second control (aqueous GO dropcasted with cycling in DMF electrolyte; blue curve) also exhibits significant redox activity 
suggesting that the reduction of the GO from the aqueous suspension is not the sole reason for the full coating and that additional GO is reduced from the DMF suspension.

Our interest in developing ERGO as an electrocatalyst support for sensing applications means that the ERGO-coated electrode must still exhibit electrochemical activity. Therefore, the response of dried $\mathrm{ERGO}_{\mathrm{DMF}}$-coated electrode towards $\mathrm{Fe}(\mathrm{CN})_{6}{ }^{3-/ 4-}$ and $\mathrm{Ru}\left(\mathrm{NH}_{3}\right)_{6}{ }^{3+/ 2+}$, two well-known redox couples for characterizing carbon electrodes, was assessed to characterize the $\mathrm{ERGO}_{\mathrm{DMF}}$ electrodes. Figure 4 compares the voltammetric response of the $\mathrm{ERGO}_{\mathrm{DMF}}$ electrode to a clean glassy carbon (GC) electrode in $1 \mathrm{mM}$ $\mathrm{Fe}(\mathrm{CN})_{6}^{3-/ 4-}($ Figure $4 \mathrm{~A})$ and $1 \mathrm{mM} \mathrm{Ru}\left(\mathrm{NH}_{3}\right)_{6}{ }^{3+/ 2+}$ (Figure 4B), both dissolved in $1 \mathrm{M} \mathrm{KCl}$, respectively. It should be first noted that both plots illustrate that the ERGO $\mathrm{DMF}_{\text {DF }}$ electrode has a much larger double-layer charging current compared to the clean glassy carbon electrode. This is expected considering the increased surface roughness and thickness of the ERGO layer, and also suggests that the ERGO layer is not very compact.[5] Additionally, these curves were not smoothed and demonstrate that the noise exhibited during the deposition of the ERGODMF film does not result in a noisy signal in an aqueous electrolyte solution.

$\mathrm{Fe}(\mathrm{CN})_{6}{ }^{3-/ 4-}$ is known to be sensitive to the surface chemistry of carbon electrodes.[25] Despite the large charging current exhibited by the ERGO ${ }_{\text {DMF }}$ electrode, a reversible redox couple is observed, shifted slightly negative compared to that on clean GC. The $\Delta \mathrm{E}_{\mathrm{p}}$ for the $\mathrm{ERGO}_{\mathrm{DMF}}$ electrode was $90 \pm 40 \mathrm{mV}$, which, as expected, was overall larger than that on clean GC $(63 \pm 3 \mathrm{mV})$. However, this still shows acceptable electrochemical 
activity for the $\mathrm{ERGO}_{\mathrm{DMF}}$ electrode. It is important to note that the $\mathrm{ERGO}_{\mathrm{DMF}}$ electrode was exposed to the laboratory atmosphere for an extended period of time and no extensive pretreatment was applied, compared to the freshly polished GC electrode. This reflects $\mathrm{ERGO}_{\mathrm{DMF}}$ 's relative resistance to fouling, a feature that will be important when incorporated into a sensor. Additionally, the observed $\Delta \mathrm{E}_{\mathrm{p}}$ compares favorably to values found by others for ERGO reduced from aqueous suspensions. For example, Molina et al. reported a $\Delta \mathrm{E}_{\mathrm{p}}$ of $121 \mathrm{mV}$ for ERGO on Pt,[10] and Guo et al. reported a $\Delta \mathrm{E}_{\mathrm{p}}$ of $80 \pm 3 \mathrm{mV}$ for ERGO on GC.[5] Similar results were presented by Chen and coworkers.[4]

Figure 4B shows the voltammetric response of $\mathrm{ERGO}_{\mathrm{DMF}}$ and clean GC electrodes towards $\mathrm{Ru}\left(\mathrm{NH}_{3}\right)_{6}{ }^{3+/ 2+}$. This is an outer-sphere redox couple that is known to be insensitive to surface chemistry, but rather sensitive to the density of electronic states in the electrode near the formal potential of the redox system, and is essentially a measure of the electrochemical conductivity. The $\Delta \mathrm{E}_{\mathrm{p}}$ value for this couple was $80 \pm 20 \mathrm{mV}$ which is larger than the $64 \pm 1 \mathrm{mV}$ exhibited by the clean GC electrode. For comparison, Molina et al. and Guo et al. observed $\Delta \mathrm{E}_{\mathrm{p}}$ values of $101 \mathrm{mV}$ and $67 \pm 1 \mathrm{mV}$ for ERGO/Pt and ERGO/GC, respectively. $[5,10]$ These results show that the ERGO $_{\text {DMF }}$ electrode possesses adequate electrochemical conductivity to serve as a support material.

\subsection{Physical \& Chemical Characterization}

While the $\mathrm{ERGO}_{\mathrm{DMF}}$ electrode clearly results in a reproducibly coated electrode that maintains its electrochemical properties, we were also interested in how the reduction conditions impacted the physical and chemical characteristics of the ERGO coating. Figure 
5 shows a microscopic comparison between the $\mathrm{ERGO}_{\mathrm{Aq}}(5 \mathrm{~A}, 5 \mathrm{C}, 5 \mathrm{E})$ and $\mathrm{ERGO}_{\mathrm{DMF}}(5 \mathrm{~B}, 5 \mathrm{D}$, $5 \mathrm{~F}$ ) electrodes. Figure $5 \mathrm{~A}$ and $\mathrm{B}$ show the optical micrographs of the $\mathrm{ERGO}_{\mathrm{Aq}}$ and $\mathrm{ERGO}_{\mathrm{DMF}}$ layers, respectively. It is clear that the $\mathrm{ERGO}_{\mathrm{DMF}}$ electrode exhibits a visually thicker, more uniform coating (in the center of the exposed electrode area) compared to the $\mathrm{ERGO}_{\mathrm{Aq}}$. This is attributed to the lack of $\mathrm{H}_{2}$ evolution in the nonaqueous reduction. Scanning electron (5C and 5D) and atomic force (5E and 5F) micrographs support the optical images. Both SEM and AFM images confirm that the $\mathrm{ERGO}_{\mathrm{DMF}}$ layer is a fuller coating that is thicker and rougher compared to the $\mathrm{ERGO}_{\mathrm{Aq}}$ deposition. $\mathrm{RMS}$ roughness for the $\mathrm{ERGO}_{\mathrm{DMF}}$ was determined to be $134 \mathrm{~nm}$ compared to $29 \mathrm{~nm}$ for the $\mathrm{ERGO}_{\mathrm{Aq}}$ layer. Interestingly, despite the better coverage and obvious increased roughness, the $\mathrm{ERGO}_{\mathrm{DMF}}$ electrode did not exhibit a higher double layer current, at $c a .-100 \mathrm{mV}$, in the $\mathrm{HCl} / \mathrm{KCl}$ experiment shown in Figure 2. The roughness of the $\mathrm{ERGO}_{\mathrm{DMF}}$ sample is similar to that reported by Zhang et al., who reported a roughness of $\sim 177 \mathrm{~nm}$ for their ERGO, however Peng et al. reported a roughness of only $5 \mathrm{~nm} .[11,14]$ It is obvious that the ERGO ${ }_{\text {DMF }}$ electrode is a very thick layer and is consistent with the high electrochemical background current observed in Figure 4.

X-ray photoelectron spectroscopy was also performed on the modified-electrode surfaces in addition to the synthesized GO. Survey and high-resolution carbon spectra are shown in Figures 6 and 7, respectively. The survey spectrum in Figure 6 shows the spectrum for each sample with the intensity normalized to the intensity of the 01 s peak at $531 \mathrm{eV}$. Normalizing the peak intensities allows for the comparison between the relative 0 1s peak and the $\mathrm{C} 1$ s peak at $285 \mathrm{eV}$ between each spectrum. It is evident from the spectra 
that both ERGO samples contain significant impurities compared to the synthesized GO. The GO spectrum shows the most disproportionate height between the carbon and oxygen signals. It is important to note that XPS is approximately $3 x$ more sensitive to oxygen than carbon. However, the approximate composition of the synthesized GO was determined to be $59 \% \mathrm{C}$ and $37 \% \mathrm{O}$, resulting in an estimated $\mathrm{C} / 0$ ratio of $c a .1 .59$. There are also small impurity peaks observed corresponding to $\mathrm{Si}(1.6 \%), \mathrm{S}(1.6 \%), \mathrm{Cl}(0.3 \%)$, and $\mathrm{N}(0.3 \%)$. These likely originate from incomplete washing during the synthesis.

The $\mathrm{ERGO}_{\mathrm{Aq}}$ spectrum shows a relative increase in the $\mathrm{C} 1 \mathrm{~s}$ peak intensity compared with the 01 s peak, consistent with the reduction of some oxygen functionalities. The sample was estimated to contain $52 \% \mathrm{C}$ and $32 \%$, corresponding to a $\mathrm{C} / 0$ ratio of 1.63 , in addition to impurity peaks for S (2.5\%), $\mathrm{Cl}(3 \%), \mathrm{N}(2 \%), \mathrm{Na}(3 \%), \mathrm{Cu}(3 \%)$, and $\mathrm{K}(2 \%)$. The ERGO $_{\text {DMF }}$ sample exhibited C 1s (73\%) and O 1s (20\%) peaks corresponding to a C/O ratio of 3.65. This is over $2 x$ higher than the $\mathrm{ERGO}_{\mathrm{Aq}}$ sample and shows that a larger amount of the oxides are reduced in the DMF protocol. However the quantitative comparison must be used with caution as there is no way of assessing or differentiating the amount of adventitious carbon in these samples. There were additionally, small impurities of Si $(0.8 \%), \mathrm{S}(0.2 \%), \mathrm{Cl}(1 \%), \mathrm{N}(2 \%), \mathrm{F}(2.5 \%)$, and $\mathrm{Na}(0.2 \%)$. It is important to note that the quantitative atomic percent values are very approximate due to probable differences in the nanometer scale structure of the two samples within the information depth $(\sim 10 \mathrm{~nm})$. If the adventitious $\mathrm{C}$ contamination is relatively low in both samples, then both deposition methods yield significant oxide reduction within the information depth; however, the overall $\mathrm{C} / \mathrm{O}$ ratio for the $\mathrm{ERGO}_{\mathrm{DMF}}$ sample is significantly higher. The 
noticeable amounts of various impurities are at least partially due to the electrolytes $\mathrm{CaCl}$ and TBATFB), and it should be noted that some impurities might bind with 0 and thus contribute to the total measured 0 signal. This could artificially increase the atomic percent of $\mathrm{O}$ in the $\mathrm{C} / \mathrm{O}$ ratio estimations.

In addition to the survey spectra, high resolution C 1s scans were performed as shown in Figure 7. Highlighted in the figure is the region due to $\mathrm{C}=\mathrm{O}(\sim 288.5 \mathrm{eV}), \mathrm{C}-\mathrm{O}$ (286.5 eV) and C-C or C-H ( $\sim 285.0 \mathrm{eV})$ groups. It is evident that the distribution of the Ccontaining functional groups varies significantly between samples, however as mentioned above, $\mathrm{C}-\mathrm{C}$ and $\mathrm{C}-\mathrm{H}$ carbon associated with the sample material cannot be distinguished from adventitious carbon. Both ERGO samples show a large decrease in the $\mathrm{C}-\mathrm{O}$ peak and subsequent increase in the $\mathrm{C}-\mathrm{C} / \mathrm{C}-\mathrm{H}$ peak indicating reduction of the GO. However, the distribution of $\mathrm{C}=\mathrm{O}$ and $\mathrm{C}-\mathrm{O}$ species differ significantly between the two reduction methods as the $\mathrm{ERGO}_{\mathrm{Aq}}$ sample has a large $\mathrm{C}=0$ peak, which is absent in the $\mathrm{ERGO}_{\mathrm{DMF}}$ spectrum. This suggests that the ERGO DMF protocol reduces more of the GO than in the aqueous protocol, likely because lower potentials can be reached in the DMF deposition and/or the increased current efficiency due to the absence of the $\mathrm{H}_{2}$ evolution reaction.

\section{Discussion}

It is generally agreed that the GO reduction process occurs at potentials lower than 0.8 to $-1.0 \mathrm{~V}$ vs. $\mathrm{Ag} / \mathrm{AgCl}$.[7, 8, 13, 15] It is important to note that on $\mathrm{Pt}$, the $\mathrm{H}_{2}$ evolution process occurs at potentials much less negative than that required to reduce the GO. This significantly impacts the current efficiency of the GO reduction. Because of this parasitic 
process, a complete coating of the electrode surface proved to be very difficult to consistently achieve from the aqueous suspension, and a minimum of 100 cycles were required to get a fully coated electrode surface as indicated by the $\mathrm{HCl} / \mathrm{KCl}$ electrochemistry. While a full coating was sometimes achieved with the $\mathrm{ERGO}_{\mathrm{Aq}}$ samples, the success rate was quite low at only $\sim 40 \%$ (5/13 electrodes). The number of CV cycles reported to be required for a complete ERGO coating is quite diverse in the literature. Guo et al. used the $\mathrm{HCl} / \mathrm{KCl}$ electrochemical analysis to also determine that 100 cycles was the minimum number required to achieve a complete ERGO layer on Pt in $0.05 \mathrm{M} \mathrm{Na}_{2} \mathrm{HPO}_{4}$.[5] Shao et al. used the disappearance of a reduction peak in the deposition to determine that 1500 cycles were required to complete the $\mathrm{GO}$ reduction in $0.1 \mathrm{M} \mathrm{Na}_{2} \mathrm{SO}_{4}$ at $50 \mathrm{mV} / \mathrm{s}$.[12] On the other hand, Molina et al. used SEM to determine that a Pt electrode could be coated by ERGO in only 40 voltammetric cycles in $0.1 \mathrm{M} \mathrm{LiClO}_{4}$, however they did not use $\mathrm{HCl} / \mathrm{KCl}$ electrochemistry to confirm the lack of pinholes.[10] Furthermore, none of these papers reported the reproducibility of these coatings.

We theorize that the onset of $\mathrm{H}_{2}$ evolution decreases the current efficiency of the GO reduction process and additionally forms $\mathrm{H}_{2}$ bubbles that subsequently remove previously reduced GO. While more cycles may help achieve a complete ERGO coating, the additional time is not desirable and will likely produce ERGO with more defects within its structure.[11] The 100 cycles to produce the $\mathrm{ERGO}_{\mathrm{Aq}}$ coating in this study took nearly $2 \mathrm{~h}$ to complete. In contrast, the nonaqueous protocol presented here only required 25 cycles, which translates to about $0.5 \mathrm{~h}$ in time. Additionally, this protocol resulted in a complete 
coating $83 \%(10 / 12$ electrodes) of the time. This is approximately double the success rate of the aqueous protocol.

As mentioned before, previous studies that incorporated drop casting an aqueous GO suspension followed by reduction in nonaqueous solutions did not verify whether complete coating of the electrode was achieved.[6, 8] However, a complete ERGO coating was confirmed here using the nonaqueous protocol. It is important to note that the complete protocol was necessary to achieve such a coating as neither of the control electrodes, including one that was similar to those prepared in earlier studies, $[6,8]$ exhibited a complete ERGO coating. This is believed to be due to either the necessary intercalated water required for the reduction reaction, $[26,27]$ or perhaps the need for GO sites to be present on the Pt surface in order to facilitate ERGO nucleation/precipitation from the DMF suspension.

The $\mathrm{Fe}(\mathrm{CN})_{6^{3-/ 4-}}$ and $\mathrm{Ru}\left(\mathrm{NH}_{3}\right)_{6^{3+/ 2+}}$ results illustrate that the ERGODMF electrode possesses adequate electrochemical activity. There is a large deviation in the $\Delta \mathrm{E}_{\mathrm{p}}$ values for both analytes, which is likely due to inconsistent thicknesses of the casted layer. Dropcasting has been reported to result in non-uniform deposition due to the agglomeration of GO upon slow evaporation of the water.[28, 29] However, spray-coating the GO suspension onto pre-heated substrates has been reported to increase the rate of solvent evaporation in order to avoid the deposition problems encountered with drop casting.[28] This will be the focus in future studies to try and optimize the resulting ERGO layer in order to minimize both the deviation in $\Delta \mathrm{E}_{\mathrm{p}}$ as well as reduce the background current. Reducing the 
background current is particularly important before implementing $\mathrm{ERGO}_{\mathrm{DMF}}$ as a practical support material in sensing applications since the large background current will likely lead to higher limits of detection.[15]

\section{Conclusion}

A new protocol for the electrochemical reduction of graphene oxide was purposed here. This protocol consisted of drop-casting a small aliquot of aqueous GO suspension on to a Pt electrode, allowing it to dry, and then cycling the potential in a nonaqueous DMF GO suspension. Cycling in a nonaqueous suspension allowed for lower cathodic potential limits without $\mathrm{H}_{2}$ generation. This resulted in a more consistently covered electrode surface, as evidenced from the $\mathrm{HCl} / \mathrm{KCl}$ electrochemistry, that was much thicker, rougher, and more reduced than that formed using the aqueous GO suspension. The ERGODMF layer still possesses good electrochemical activity despite a rather large presence of impurities in the sample. Minimizing the impurity level of the $\mathrm{ERGO}_{\mathrm{DMF}}$ as well as the electrochemical background current through spray-coating and/or more negative reduction potentials will be investigated to further improve the ERGO coating. Optimizing the ERGO coating by improving this new protocol is expected to decrease the amount of defects, reduce the oxygen content, increase the $\mathrm{sp}^{2}$ character and conductivity, as well as improve the overall integrity of the ERGO. This in addition to its ease, speed, and reproducibility will make this attractive for practical applications, including as an electrocatalyst support for sensing applications. 


\section{Acknowledgements}

The authors would like to acknowledge Penn State Erie, The Behrend College as well as an award from the National Science Foundation (CMI; grant CHE-1305660) for the financial support for this work. The assistance of Vince Bojan (XPS) and Timothy Tighe (microscopy) at the Penn State University Materials Characterization Laboratory is greatly appreciated. The proof reading of this manuscript by Susanne Simkovitch is greatly appreciated.

\section{References}

[1] S.Y. Toh, K.S. Loh, S.K. Kamarudin, W.R.W. Daud, Graphene Production via Electrochemical Reduction of Graphene Oxide: Synthesis and Characterisation, Chem. Eng. J., 251 (2014) 422.

[2] H.-L. Guo, X.-F. Wang, Q.-Y. Qian, F.-B. Wang, X.-H. Xia, A Green Approach to the Synthesis of Graphene Nanosheets, ACS Nano, 3 (2009) 2653.

[3] Y. Wu, M. Lv, B. Li, J. Ge, L. Gao, A Rapid, Green and Controllable Strategy to Fabricate Electrodeposition of Reduced Graphene Oxide Film as Sensing Materials for Determination of Taxifolin, Nano, 10 (2015) 1550044.

[4] L. Chen, Y. Tang, K. Wang, C. Liu, S. Luo, Direct electrodeposition of reduced graphene oxide on glassy carbon electrode and its electrochemical application, Electrochem. Commun., 13 (2011) 133.

[5] S.-X. Guo, S.-F. Zhao, A.M. Bond, J. Zhang, Simplifying the Evaluation of Graphene Modified Electrode Performance Using Rotating Disk Electrode Voltammetry, Langmuir, 28 (2012) 5275.

[6] Y. Harima, S. Setodoi, I. Imae, K. Komaguchi, Y. Ooyama, J. Ohshita, H. Mizota, J. Yano, Electrochemical reduction of graphene oxide in organic solvents, Electrochim. Acta, 56 (2011) 5363. 
[7] M. Hilder, B. Winther-Jensen, D. Li, M. Forsyth, D.R. MacFarlane, Direct electrodeposition of graphene from aqueous suspensions, Phys. Chem. Chem. Phys., 13 (2011) 9187.

[8] J. Kauppila, P. Kunnas, P. Damlin, A. Viinikanoja, C. Kvarnström, Electrochemical reduction of graphene oxide films in aqueous and organic solutions, Electrochim. Acta, 89 (2013) 84.

[9] C. Liu, K. Wang, S. Luo, Y. Tang, L. Chen, Direct Electrodeposition of Graphene Enabling the One-Step Synthesis of Graphene-Metal Nanocomposite Films, Small, 7 (2011) 1203.

[10] J. Molina, J. Fernández, C. García, A.I. del Río, J. Bonastre, F. Cases, Electrochemical characterization of electrochemically reduced graphene coatings on platinum. Electrochemical study of dye adsorption, Electrochim. Acta, 166 (2015) 54.

[11] X.-Y. Peng, X.-X. Liu, D. Diamond, K.T. Lau, Synthesis of electrochemically-reduced graphene oxide film with controllable size and thickness and its use in supercapacitor, Carbon, 49 (2011) 3488.

[12] Y. Shao, J. Wang, M. Engelhard, C. Wang, Y. Lin, Facile and controllable electrochemical reduction of graphene oxide and its applications, J. Mater. Chem., 20 (2010) 743.

[13] A. Viinikanoja, Z. Wang, J. Kauppila, C. Kvarnstrom, Electrochemical reduction of graphene oxide and its in situ spectroelectrochemical characterization, Phys. Chem. Chem. Phys., 14 (2012) 14003.

[14] X. Zhang, D. Zhang, Y. Chen, X. Sun, Y. Ma, Electrochemical reduction of graphene oxide films: Preparation, characterization and their electrochemical properties, Chin. Sci. Bull., 57 (2012) 3045.

[15] Z. Zhang, J. Yan, H. Jin, J. Yin, Tuning the reduction extent of electrochemically reduced graphene oxide electrode film to enhance its detection limit for voltammetric analysis, Electrochim. Acta, 139 (2014) 232.

[16] M. Zhou, Y. Wang, Y. Zhai, J. Zhai, W. Ren, F. Wang, S. Dong, Controlled Synthesis of Large-Area and Patterned Electrochemically Reduced Graphene Oxide Films, Chem.-Eur. J., 15 (2009) 6116. 
[17] S.-J. Li, Y. Xing, D.-H. Deng, M.-M. Shi, P.-P. Guan, A comparative study of different types of reduced graphene oxides as electrochemical sensing platforms for hydroquinone and catechol, J. Solid State Electrochem., 19 (2015) 861.

[18] F. Wang, Y. Cao, B. Zhang, L. Gao, J. Ge, A Sensitive Amperometric Sensor for the Determination of Sophocarpine Based on Vertically Oriented Graphene Nanosheets Modified Glassy Carbon Electrode, J. Electrochem. Soc., 162 (2015) H352.

[19] S.L. Ting, C.X. Guo, K.C. Leong, D.-H. Kim, C.M. Li, P. Chen, Gold nanoparticles decorated reduced graphene oxide for detecting the presence and cellular release of nitric oxide, Electrochim. Acta, 111 (2013) 441.

[20] D.C. Marcano, D.V. Kosynkin, J.M. Berlin, A. Sinitskii, Z. Sun, A. Slesarev, L.B. Alemany, W. Lu, J.M. Tour, Improved synthesis of graphene oxide, ACS Nano, 4 (2010) 4806.

[21] J.I. Paredes, S. Villar-Rodil, A. Martínez-Alonso, J.M.D. Tascón, Graphene Oxide Dispersions in Organic Solvents, Langmuir, 24 (2008) 10560.

[22] D.T. Sawyer, A. Sobkowiak, J.L. Roberts Jr., Solvents and Electrolytes, in: Electrochemistry for Chemists, John Wiley \& Sons, New York, 1995, pp. 505.

[23] J. Zhang, B.P. Ting, M. Khan, M.C. Pearce, Y. Yang, Z. Gao, J.Y. Ying, Pt Nanoparticle Label-Mediated Deposition of Pt Catalyst for Ultrasensitive Electrochemical Immunosensors, Biosens. Bioelectron., 26 (2010) 418.

[24] D.T. Sawyer, A. Sobkowiak, J.L. Roberts Jr., Hydronium Ions $\left(\mathrm{H}_{3} \mathrm{O}^{+}\right)$, Bronsted Acids $(\mathrm{HA})$, and Molecular Hydrogen $\left(\mathrm{H}_{2}\right)$, in: Electrochemistry for Chemists, John Wiley \& Sons, New York, 1995, pp. 505.

[25] P. Chen, R.L. McCreery, Control of Electron Transfer Kinetics at Glassy Carbon Electrodes by Specific Surface Modification, Anal. Chem., 68 (1996) 3958.

[26] M. Acik, C. Mattevi, C. Gong, G. Lee, K. Cho, M. Chhowalla, Y.J. Chabal, The Role of Intercalated Water in Multilayered Graphene Oxide, ACS Nano, 4 (2010) 5861.

[27] D. Kim, D.W. Kim, H.-K. Lim, J. Jeon, H. Kim, H.-T. Jung, H. Lee, Intercalation of Gas Molecules in Graphene Oxide Interlayer: The Role of Water, J. Phys. Chem. C, 118 (2014) 11142. 
[28] S. Gilje, S. Han, M. Wang, K.L. Wang, R.B. Kaner, A Chemical Route to Graphene for Device Applications, Nano Lett., 7 (2007) 3394.

[29] G. Eda, M. Chhowalla, Chemically Derived Graphene Oxide: Towards Large-Area ThinFilm Electronics and Optoelectronics, Adv. Mater., 22 (2010) 2392. 


\section{Figure Captions}

Figure 1. Voltammetric $i-E$ curves for the electrochemical reduction of graphene oxide in (A) aqueous and (B) DMF GO suspensions. Scan rate for both was 50 $\mathrm{mV} / \mathrm{s}$. Numbers denote the cycle number in the deposition.

Figure 2. Voltammetric $i$ - $E$ curves for $5 \mathrm{mM} \mathrm{HCl}$ in $0.5 \mathrm{M} \mathrm{KCl}$ at clean Pt (dotted black), $\mathrm{ERGO}_{\mathrm{DMF}}$ (dashed red), and $\mathrm{ERGO}_{\mathrm{Aq}}$ (solid blue) electrodes. Inset shows enlarged curves for only $\mathrm{ERGO}_{\mathrm{DMF}}$ and $\mathrm{ERGO}_{\mathrm{Aq}}$ electrodes to highlight their differences. Scan rate $=50 \mathrm{mV} / \mathrm{s}$.

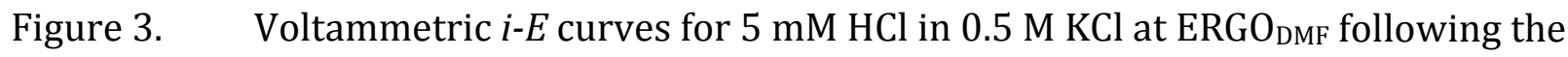
full protocol (black), Control 1 that attempted to directly reduced GO from the DMF suspension without initial drop-casting (red), and Control 2 that used only the drop-casted aqueous suspension followed by cycling in TBATFB/DMF electrolyte solution (blue). Scan rate $=50 \mathrm{mV} / \mathrm{s}$.

Figure 4. Voltammetric $i-E$ curves for $1 \mathrm{mM}(\mathrm{A}) \mathrm{Fe}(\mathrm{CN})_{6}^{3-/ 4-}$ and (B) $\mathrm{Ru}\left(\mathrm{NH}_{3}\right)_{6} 6^{3+/ 2+}$ both dissolved in $1 \mathrm{M} \mathrm{KCl}$ at ERGO $\mathrm{DMF}_{\mathrm{DF}}$ (solid red) and freshly polished glassy carbon (dotted black) electrodes. Scan rate $=50 \mathrm{mV} / \mathrm{s}$.

Figure 5. Optical (A and B), scanning electron (C and D), and atomic force ( $\mathrm{E}$ and $\mathrm{F}$ ) micrographs of $\mathrm{ERGO}_{\mathrm{Aq}}(\mathrm{A}, \mathrm{C}$, and $\mathrm{E})$ and $\mathrm{ERGO}_{\mathrm{DMF}}(\mathrm{B}, \mathrm{D}$, and $\mathrm{F})$ electrodes.

Figure 6. X-ray photoelectron spectra of synthesized G0, ERGO samples. The intensity of each spectrum has been normalized using the $01 \mathrm{~s}$ line intensity.

Figure 7. High-resolution C 1s X-ray photoelectron spectra of synthesized GO (solid black), $\mathrm{ERGO}_{\mathrm{Aq}}$ (dotted blue), and $\mathrm{ERGO}_{\mathrm{DMF}}$ (dashed red) samples. 

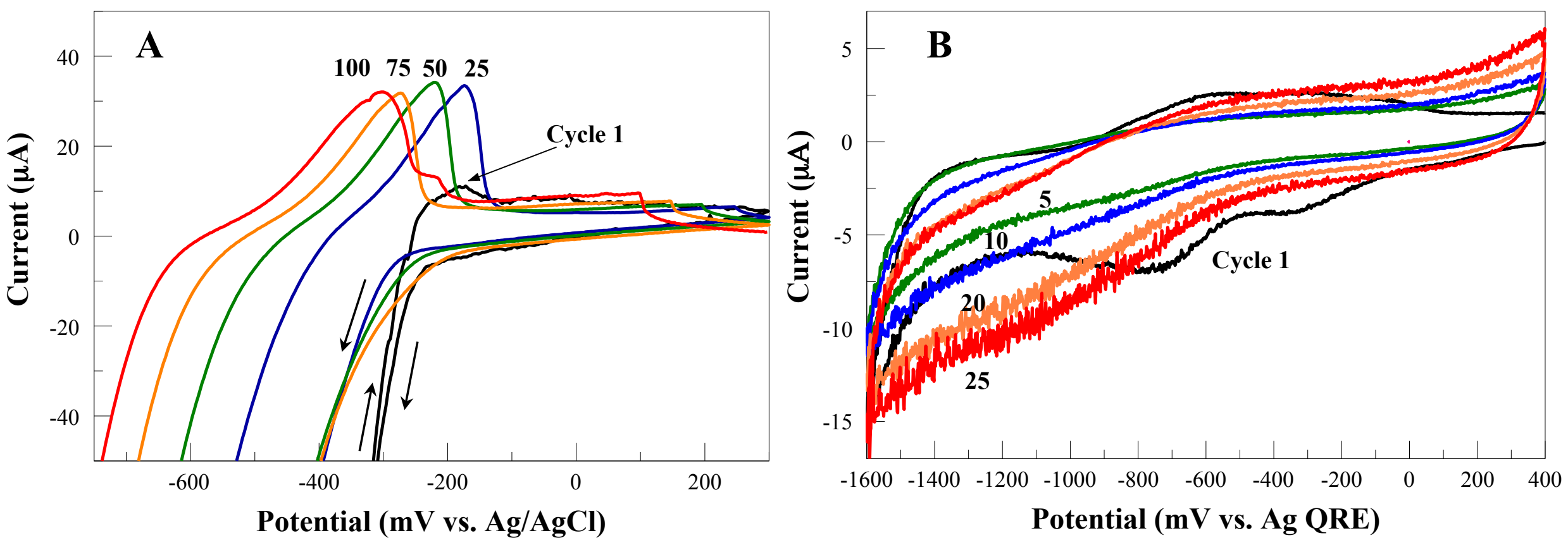

Figure 1 


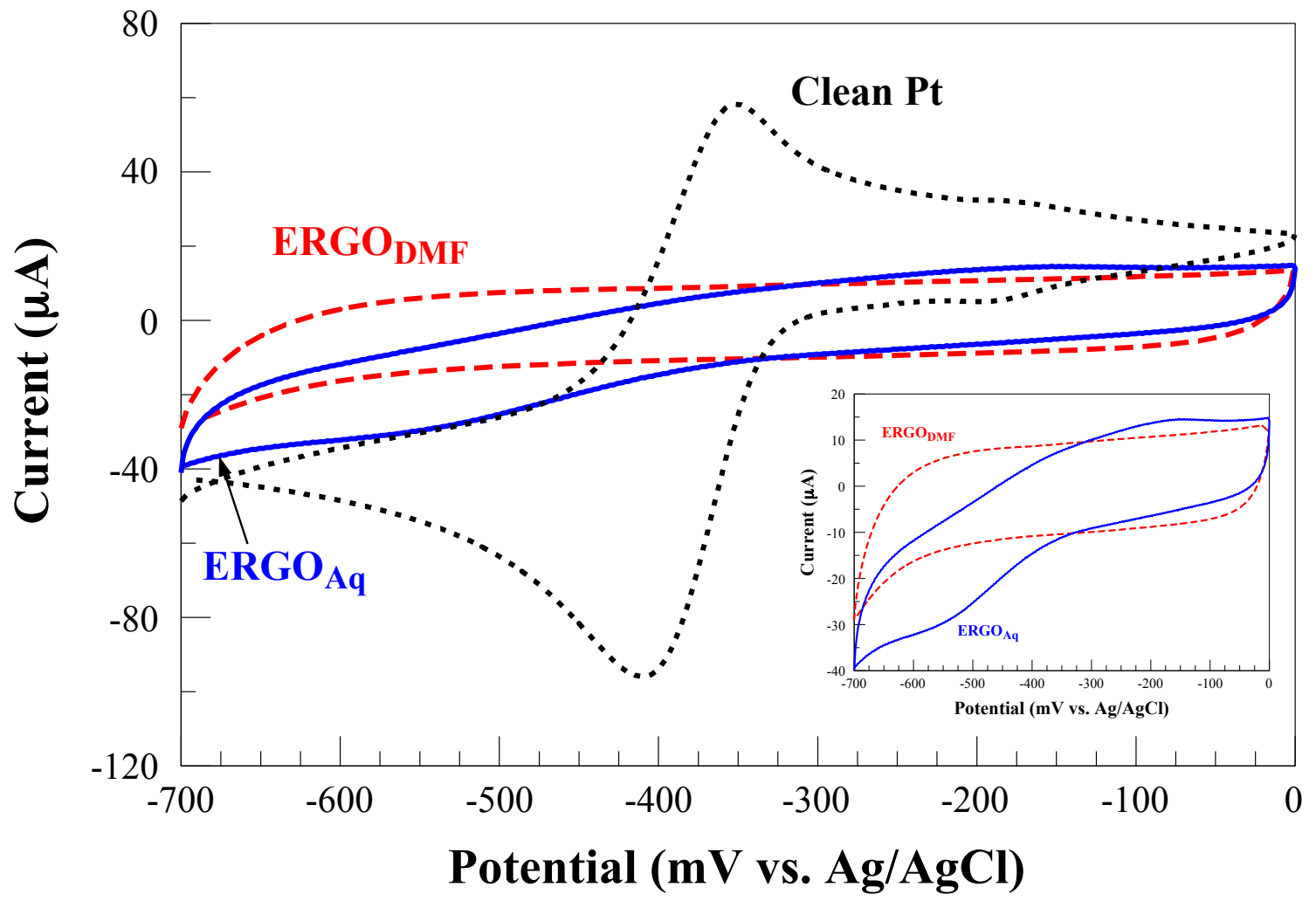

Figure 2 


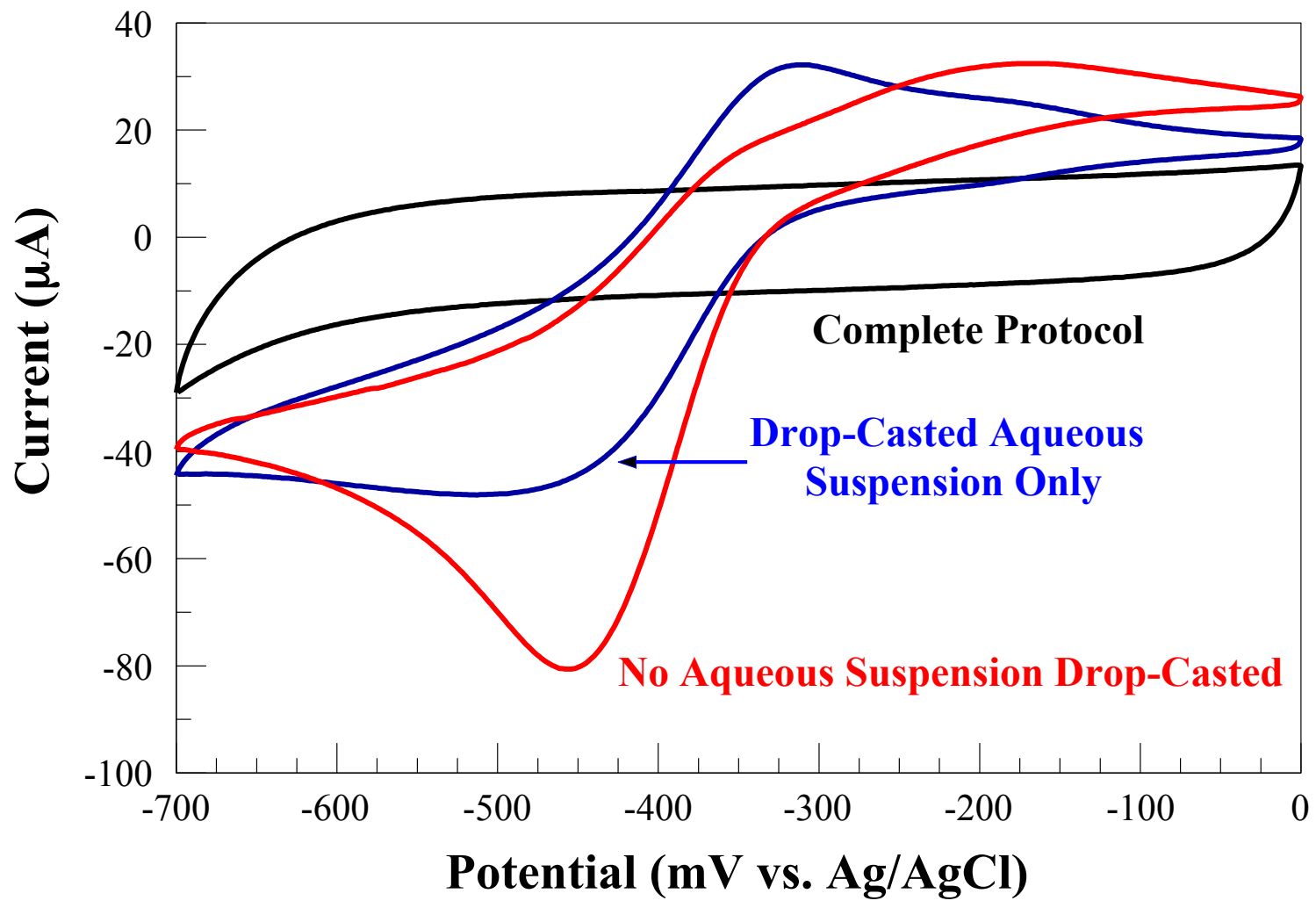

Figure 3 

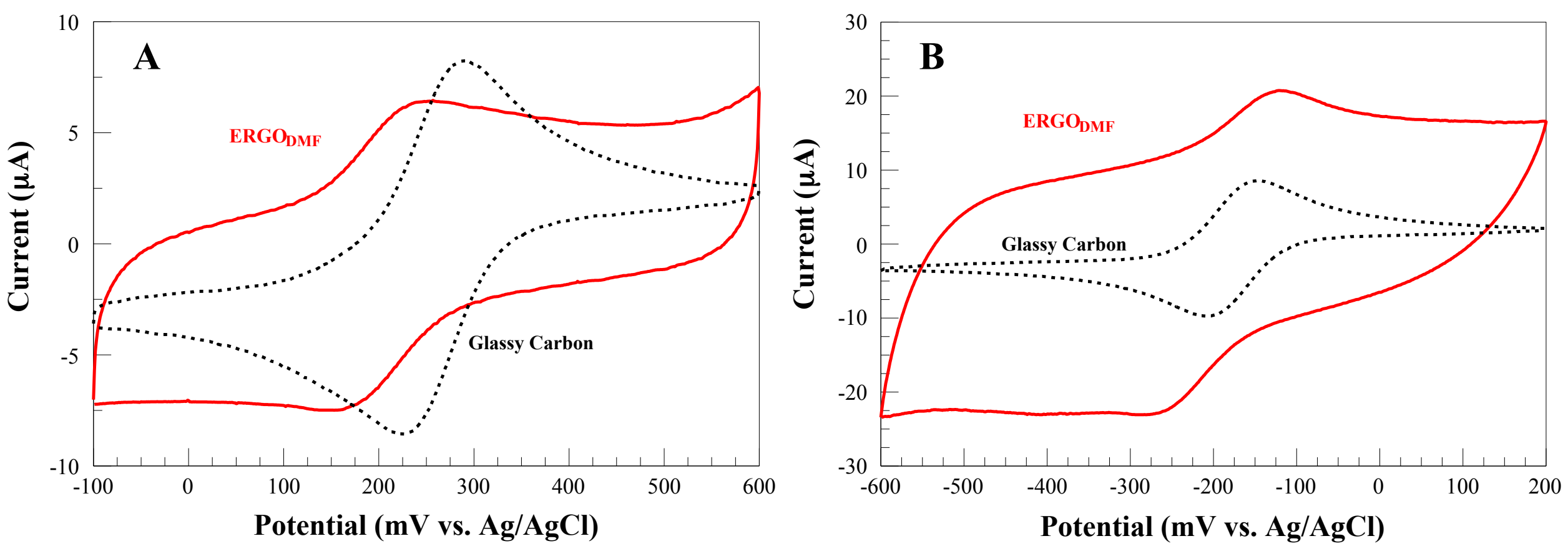

Figure 4 


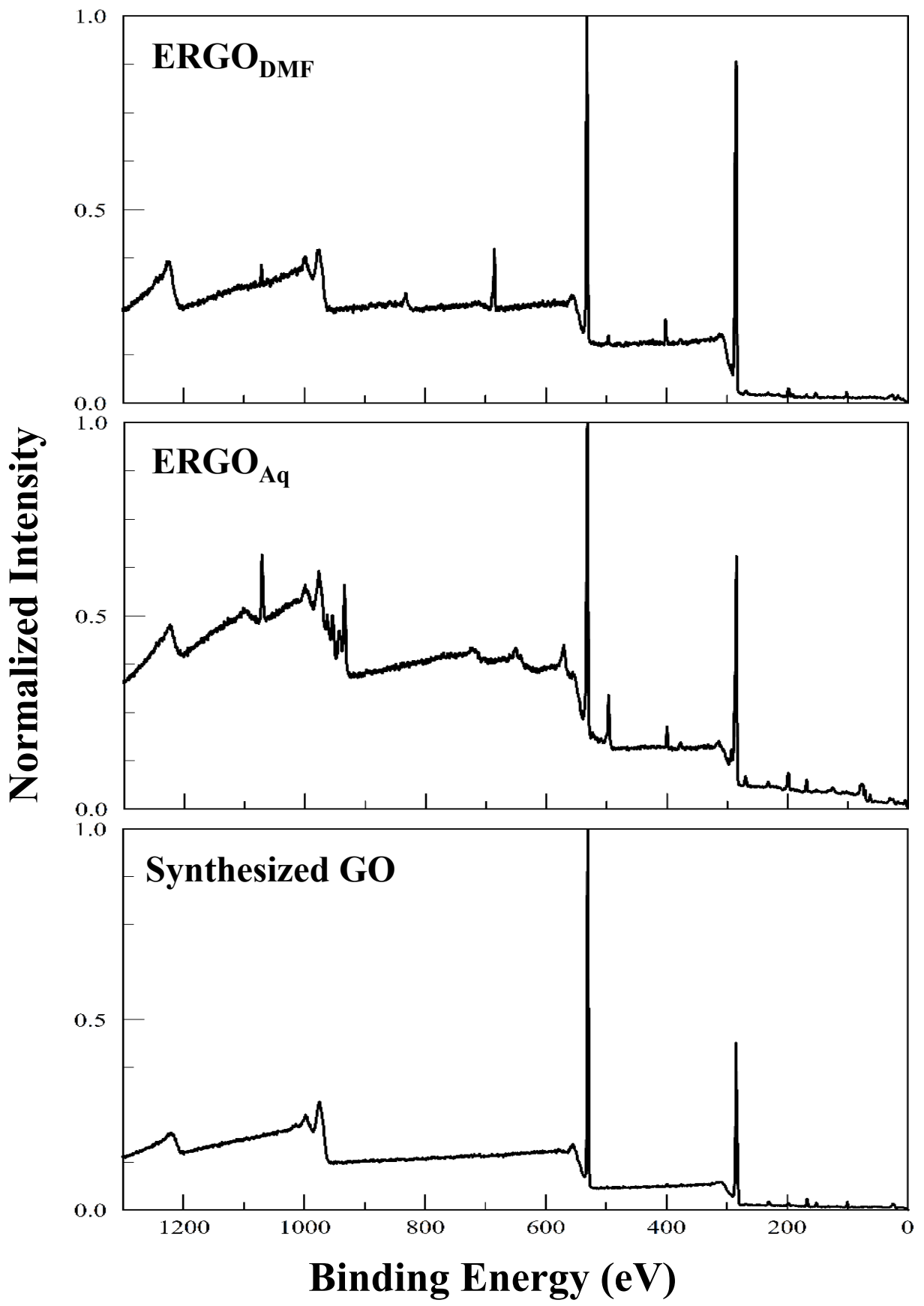

Figure 6 


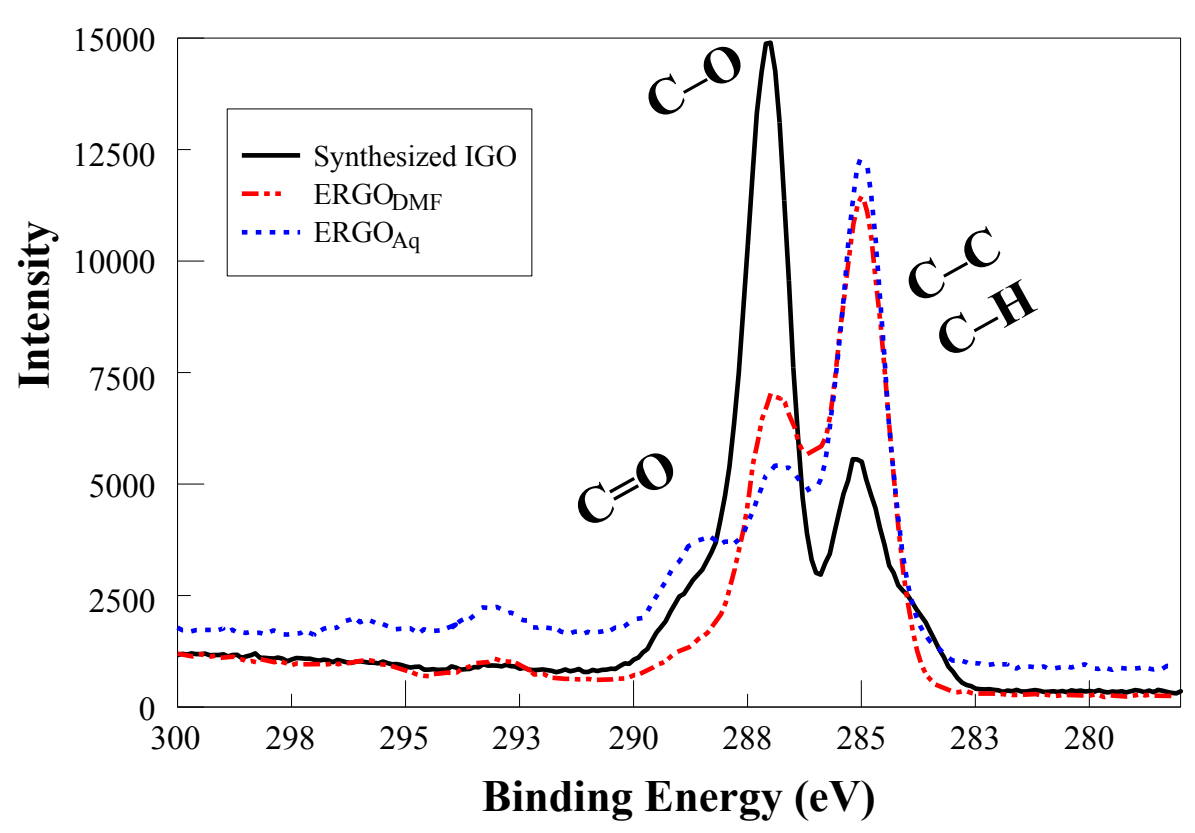

Figure 7 
Aqueous Deposition - Incomplete coating

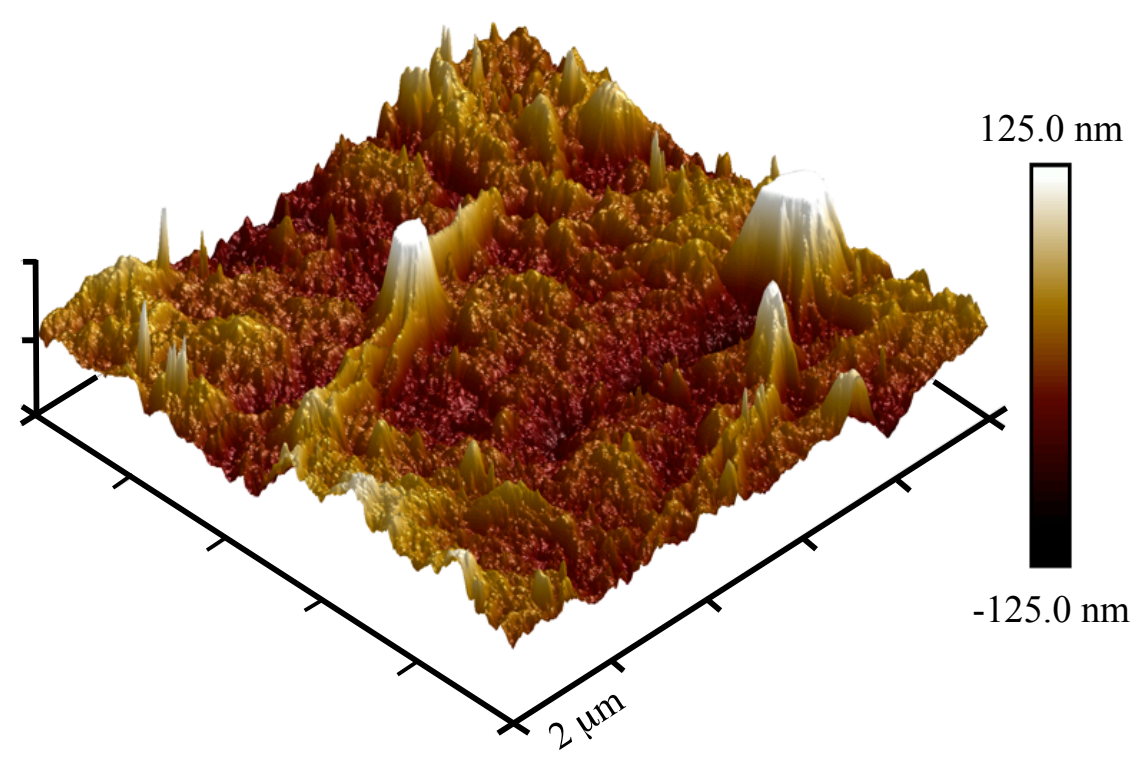

Nonaqueous Deposition - Complete coating

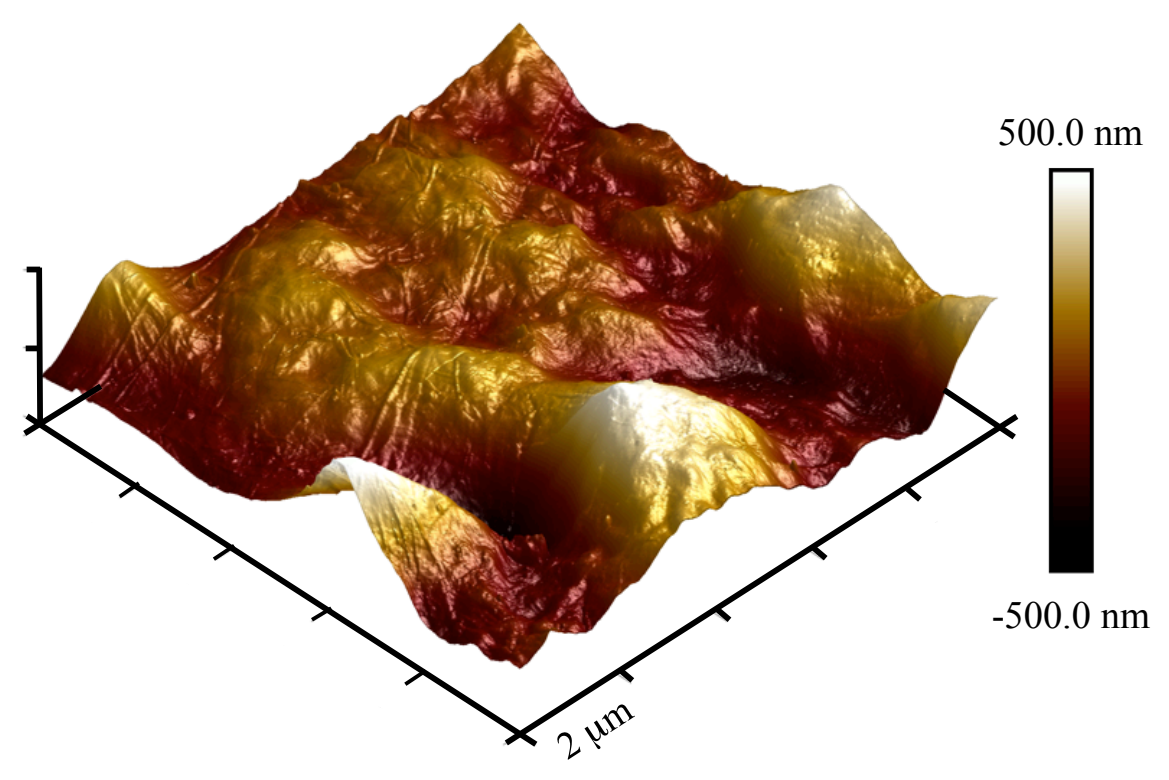

